(Aus dem physiologischen Institut der Universität Zürich.)

\title{
Die geschlechtlichen Unterschiede in der Leber des Frosehes.
}

Von

Alice Ganle.

(Hierzu Tafel I.)

Während einer Reihe von Jahren, in der ich wieder und immer wieder Gelegenheit hatte, normale Frösche zu beobachten, fielen mir Unterschiede in der Grösse der verschiedenen Organe immer deutlicher auf. Es kam mir vor, dass die Leber der Frösche zu verschiedenen Jahreszeiten nicht nur in der Grösse, sondern auch in ihrer Farbe auffallende Veränderungen zeigte. Nun sind Männchen und Weibchen ausserlich in Allem bis auf die etwas vollere Form des Körpers des Weibchens und die Daumenwarzen des Männchens nicht $\mathrm{zu}$ unterseheiden. Aber auch hier meinte ich einen Unterschied in der Grösse und Farbe der Leber bemerkt zu haben. Es reizte mich, zu erfahren, ob diese Beobachtungen nur zufällige, individuelle Verschiedenheiten waren, oder ob das veränderte Aussehen der Leber die Folge einer bestimmten Ordnung oder eines bestimmten Gesetzes des Stoffwechsels war.

\section{If ethode.}

Für diese Reihe von Versuchen wählte ich Rana esculenta, und meine Methode war hauptsächlich die des einfachen Wägens. Nachdem die Frösche direct von ihrem Behälter im Keller gebracht waren, wurden sie möglichst bald gewogen, und in gewöhnlicher Weise mit einem Scheerenschnitt und Ausbohren des Rückenmarkes getödtet. Die Thiere wurden dann secirt, irgend welche charakteristische Erscheinungen notirt und die Organe gewogen. Der Behälter im Keller hatte schiefe cementirte Wände mit eingelegten

E. Pfiñ ger, Archiv für Physiologie. Bd. 84. 
Steinen. Unten war Wasser, so dass die Frösche nass oder trocken sitzen konnten nach ihrer Wahl. Auf diese Weise habe ich innerhalb eines Jahres die Lebern von 305 Fröschen gewogen. Von diesen waren 186 Wei chen, 119 Männchen. Die Vertheilung auf die verschiedenen Monate ist folgende:

\begin{tabular}{|c|c|c|c|c|}
\hline & $q$ & $\delta$ & & q \\
\hline Januar & 13 & 8 & Juli & 25 \\
\hline Februar & 16 & 13 & August & 16 \\
\hline März & 22 & 11 & September & 13 \\
\hline April & 6 & 6 & October & 15 \\
\hline lai & 11 & 9 & November & 11 \\
\hline uni & 24 & 16 & December & 14 \\
\hline
\end{tabular}

In etwa der Hälfte der Fälle wurden die Lebern in Erhärtungsflüssigkeit eingelegt zum histologischen Studium.

\section{Die Resultate.}

Da ich in der Wahl des Materials beschränkt war, wurde ich gewungen, Frösche von sehr verschiedenem Gewicht und von verschiedenen Fundorten zu meinem Zweck zu benutzen. Ich bemerkte bald, dass Frösche, die von dem nördlichen Fusse des Uetlibergs herstammten, sich bezüglich der Entwicklung ihrer Geschlechtsorgane anders verhielten wie die von Wetzikon auf der anderen Seite des Zürichbergs, wo das Land ziemlich flach und offen ist. Dies vorausschickend gebe ich zunächst auf Tafel I Fig. 1 das relative Gewicht der Leber zum Körpergewicht, d. b. ich habe das Lebergewicht durch das Körpergewicht dividirt. Die Abscissen geben die Tage an, die Ordinaten die Gewichte, und zwar entspricht je $1 \mathrm{~mm}$ $1 \mathrm{mg}$. Die gezogenen Linien verbinden die Gewichte der Weibchen, die punktirten die der Männchen. Eine merkwürdig zackige Linie bietet diese Curve uns dar, und auf den ersten Blick denkt man, da hat man die individuellen Versehiedenheiten in Hülle und Fülle. Aber genauer betrachtet ist es sehr deutlich, dass diese individuellen Verschiedenheiten sich innerhalb ziemlich bestimmter Grenzen bewegen, und dass die Curve des Jahres eine fast S-förmig gebogene Linie bildet. Ausserdem liegt die Linie der Männchen in der Mehrzahl der Fälle über der der Weibchen. Ein anderer Gedanke, der mich bei dem Studium dieser Curve beschäftigte, war, ob dann nicht 

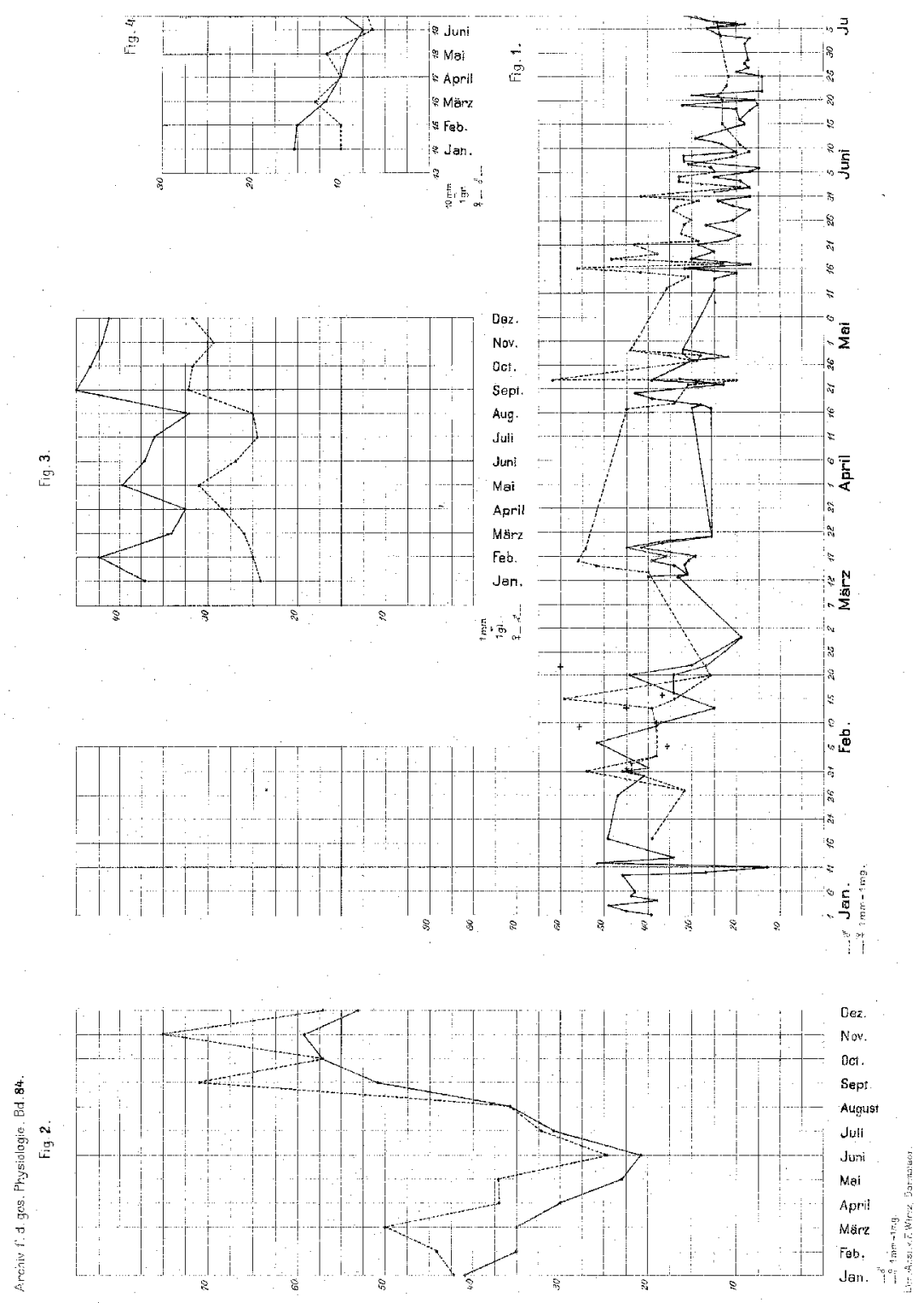

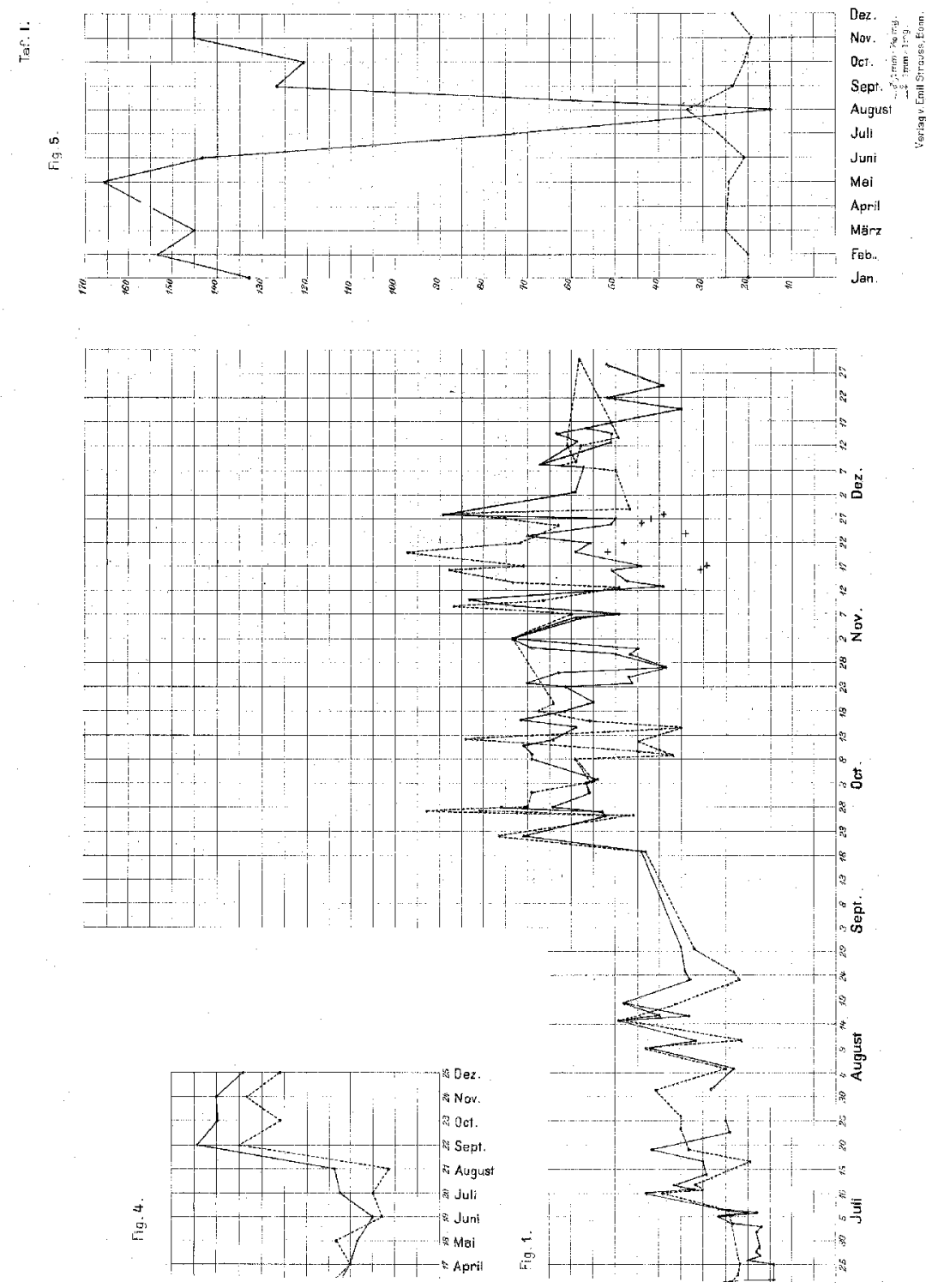
einfach der grössere Frosch die grössere Leber habe, auch wenn man nur das Verhältniss des Gewichts berücksichtigt. Wenn man auf der Tafel die Kreuze bemerkt, die das Gewicht der betreffenden Frösche in Grammen ...ssdrücken, muss man verneinen, dass eine Regel in dieser Beziehung sich leicht ausfindig machen lässt.

Dieser Gedanke wird geprüft durch die Curve, die in Fig. 2 dargestellt ist. Hier sind die Durchschnittsgewichte der Leber für den Monat durch die Durchschnittsgewichte der Frösche dividirt und sonst wie in Tafel I Fig. 1 ausgführt. Wenn man einen Durchschnitt für den Monat hat, so sind darin sowohl grössere wie kleinere Frösche enthalten, und man wird von der Grösse des einzelnen Thieres unabhängig. Die Curven deuten an, dass sowohl bei Männchen wie bei Weibchen die Gewichte der Leber von Monat zu Monat grösseren Schwankungen unterliegen. Diese Differenz muss also nicht bloss von der Grösse des einzelnen Thieres abhängen, sie muss Ursachen haben, die allen Fröschen gemeinschaftlich sind. Dass diese theilweise von der kälteren oder wärmeren Jahreszeit, von Hunger- und Fressperiode abbängen, ist kaum zu bezweifeln, wenn man diese Figur im Verhältniss zu den Monaten ansieht. Aher wenn kein anderes Moment in Betracht käme, würden. Männchen und Weibchen ganz ähnliche Curven zeigen. Wir sehen nun in unserer Curve, dass dies nicht der Fall ist. Was kann nun einen Einfluss haben, der für Männchen und Weibchen nicht derselbe ist? Es ist ein anderes wichtiges Moment im Leben des Frosches, der jährliche Aufbau der Geschlechtsproducte und das Laichen. Dieses letztere geschieht, je nach der Witterung, im Mai, Juni oder Juli. Dieses Jahr fand man grosse Fierstöcke bis zum 10. Juli. Diesen Thatsachen entsprechend sehen wir die Lebercurve herabsinken bis Juli und dann sich allmälig heben, bis sie ein dreifaches Lebergewicht beim Männchen, ein zweifaches beim Weibchen andeutet. Theilweise rühren wohl die grösseren Gewichte der Leber im Spätsommer und Herbst von der reichlicheren Nahrung und dem neuen Wachsthume der Frösche her. Nun sind auf Tafel I Fig. 3 die Durchschnittsgewichte der Frösche angegeben. Man sieht, dass wir wenigstens im Herbst schwerere Frösche bekamen als im Frühjahr und Sommer. Ferner ist es deutlich aus dieser Curve zu ersehen, dass die Leber der Männchen viel grösser ist im Verhältniss zum Körpergewicht wie die der Weibchen, denn auf Tafel I Fig. 1 laufen die Lebergewichte der Weibchen fast das ganze Jahr durch unter denen der Männchen, 
während Fig. 3 darstellt, wie die gesammten Weibchen oder die lebenden Weibchen das ganze Jahr durch weit schwerer waren wie die entsprechenden Männchen.

Nunmehr gibt Tafe I Fig. 4 die einfachen Durchschnittsgewichte der Leber während des Jahres an. Die Leber der Weibchen steht nicht im Verhältniss, sondern ist absolut genommen schwerer wie die der Männchen, mit Ausnahme von den drei Monaten März, April, Mai. Warum sinkt wohl das Lebergewicht so tief in den drei obengenannten Monaten? Werfen wir einen Blick auf Tafel I Fig. 5, die eine Curve der Gewichte der Geschlechtsproducte zeigt - die Eierstöcke sind pro Gramm Gewicht umgerechnet -, und wie wir finden, dass die Eierstöcke im Mai sehr gross werden, und wie wir eben gesehen haben, wird gleichzeitig die Leber sehr klein. Nachdem die Laichzeit vorbei ist, erst dann wird die Leber der Weibchen grösser. Beim Männchen ist das Verhältniss anders. Auch hier sind die Curven der Leber und der Geschlechtsproducte im Juni fast am tiefsten, aber wie wir aus der Arbeit von Ploetz ${ }^{1}$ ) wissen, haben die Hoden der Esculenta keinen so eharakteristischen Cyklus wie der eben angedeutete der Eierstöcke. Nach ihm, wie nach meiner Curve, sind die Hoden im Juli und August etwas grösser wie sonst. $\mathrm{Ob}$ die Leber auch eine Beziehung zu dem Aufbau der Hoden hat, müssen wir einstweilen unentschieden sein lassen, doch sowohl aus Fig. 2 als aus Fig. 4 sehen wir, dass sie erst grösser wird im September zu der Zeit, wo die Hoden wieder kleiner geworden sind.

Das histologisehe Bild sollte uns über diese Beziehungen etwas Aufklärung geben, aber das Material ist so gross, und merkwürdiger Weise zeigt sich die Structur der normalen Frösche so unendlich mannigfaltig, dass es noch zu früh ist, genauer darauf einzugehen. Hier möchte nur erwähnt werden, dass die Lebern der Weibchen im Mai und Juni mit einzelnen Ausnahmen ganz anders aussehende Zellen haben als die der Männchen. Der Untersehied besteht in der Grösse der Zellen, die sehr klein sind, und im Protoplasma, das homogen ist, während bei den Männchen die Zellen grösser sind und das Protoplasma sich ungleichmässig färbt. Bemerkẻnswerth ist ferner, dass auch bei den Lebern der Männchen diese Verände-

1) A. J. Ploetz: Die Vorgänge in den Froschhoden unter dem Einfluss der Jahreszeit. Arch. f. Anat. u. Physiol. Supplement-Bd. 1890. 
rung auftritt, d. h. die Zellen werden klein, das Protoplasma homogen, aber das geschieht erst später, vereinzelt im Juni, häufig im Juli.

Zum Schluss möchte ich noch ein Mal hervorheben, wie man einen Unterschied mac en muss zwischen dem absoluten Gewicht der Leber und dem Verhältniss dieses Gewichtes zu dem Körpergewicht. Während das erstere mit den Ausnahmen der obengenannten drei Monate bei den Weibchen grösser ist als bei den Männchen, sind die relativen Lebergewichte das ganze Jahr hindurch bei den Männchen grössere. Diese Differenz hat aber eine Begründung in den geschlechtlichen Verhältnissen, nicht nur weil sie Männchen und Weibchen trennt, sondern auch desshalb, weil bei den Weibchen die Veränderungen in den Gewichten der Leber synchron verlaufen mit den Veränderungen in den Gewichten der Geschlechtsorgane. 